\title{
Genome-Based Comparison of Clostridioides difficile: Average Amino Acid Identity Analysis of Core Genomes
}

\author{
Adriana Cabal ${ }^{1} \cdot$ Se-Ran Jun ${ }^{2} \cdot$ Piroon Jenjaroenpun $^{2} \cdot$ Visanu Wanchai $^{2} \cdot$ Intawat Nookaew $^{2}$. \\ Thidathip Wongsurawat ${ }^{2} \cdot$ Mary J. Burgess $^{3} \cdot$ Atul Kothari $^{3} \cdot$ Trudy M. Wassenaar $^{1,2} \cdot$ David W. Ussery $^{2}$
}

Received: 30 August 2017 / Accepted: 2 February 2018 / Published online: 14 February 2018

(C) The Author(s) 2018. This article is an open access publication

\begin{abstract}
Infections due to Clostridioides difficile (previously known as Clostridium difficile) are a major problem in hospitals, where cases can be caused by community-acquired strains as well as by nosocomial spread. Whole genome sequences from clinical samples contain a lot of information but that needs to be analyzed and compared in such a way that the outcome is useful for clinicians or epidemiologists. Here, we compare 663 public available complete genome sequences of $C$. difficile using average amino acid identity (AAI) scores. This analysis revealed that most of these genomes $(640,96.5 \%)$ clearly belong to the same species, while the remaining 23 genomes produce four distinct clusters within the Clostridioides genus. The main $C$. difficile cluster can be further divided into sub-clusters, depending on the chosen cutoff. We demonstrate that MLST, either based on partial or full gene-length, results in biased estimates of genetic differences and does not capture the true degree of similarity or differences of complete genomes. Presence of genes coding for $C$. difficile toxins A and B (ToxA/B), as well as the binary $C$. difficile toxin (CDT), was deduced from their unique PfamA domain architectures. Out of the 663 C. difficile genomes, 535 (80.7\%) contained at least one copy of ToxA or ToxB, while these genes were missing from 128 genomes. Although some clusters were enriched for toxin presence, these genes are variably present in a given genetic background. The CDT genes were found in 191 genomes, which were restricted to a few clusters only, and only one cluster lacked the toxin A/B genes consistently. A total of 310 genomes contained ToxA/B without CDT (47\%). Further, published metagenomic data from stools were used to assess the presence of $C$. difficile sequences in blinded cases of $C$. difficile infection (CDI) and controls, to test if metagenomic analysis is sensitive enough to detect the pathogen, and to establish strain relationships between cases from the same hospital. We conclude that metagenomics can contribute to the identification of CDI and can assist in characterization of the most probable causative strain in CDI patients.
\end{abstract}

Keywords $C$. difficile $\cdot$ AAI $\cdot$ MLST $\cdot$ Community-acquired infections $\cdot$ Comparative genomics

Electronic supplementary material The online version of this article (https://doi.org/10.1007/s00248-018-1155-7) contains supplementary material, which is available to authorized users.

David W. Ussery

DWUssery@uams.edu

1 Molecular Microbiology and Genomics Consultants, Tannenstrasse 7, 55576 Zotzenheim, Germany

2 Arkansas Center for Genomic Epidemiology and Medicine, Department of Biomedical Informatics, University of Arkansas for Medical Sciences, 4301 W. Markham Str., Slot 782, Little Rock, AR 72205, USA

3 Division of Infectious Diseases, University of Arkansas for Medical Sciences, Little Rock, AR 72205, USA

\section{Introduction}

Clostridioides difficile ( $C$. difficile) is a Gram-positive, anaerobic bacillus that is responsible for pseudomembranous colitis; it is also a common cause of nosocomial diarrhea, conditions whose morbidity and mortality have dramatically increased in the past decade [1-3]. Nosocomial infections caused by $C$. difficile are a recurrent problem and increasingly young individuals are recognized as being at high risk, in contrast with the historical $C$. difficile incidence trends, which pointed to elderly hospitalized patients mainly [4]. In the traditional view, hospitalized patients are exposed to the spores of $C$. difficile by direct contact with medical staff, via contaminated utensils or from the hospital environment, although 
food can also be involved in the transmission process [5]. In patients with an effective immune response, colonization of the gut by $C$. difficile can occur without the presentation of clinical signs. However, in individuals with a history of repeated exposure to certain antibiotics, those who are immunocompromised or suffer from underlying enteric diseases, C. difficile can dramatically increase in numbers [6]. This has knock-on effects on the gut microbiome and results in a disbalance of other bacterial species (dysbiosis), resulting in a semi-permanently altered gut micro-environment. Once this condition is established, treatment becomes very difficult. In such cases, a fecal transplant may be the only option, which has shown efficacy rates as high as $90 \%[7,8]$.

The taxonomic description of the genus Clostridium, to which $C$. difficile used to belong, has undergone multiple revisions over the years. The genus expanded and was split up again, resulting in some confusion about its members. There have been at least 240 bacterial species that, at some time in the past, were accepted as a member of Clostridium. In 1994, a major revision of the genus was proposed, which moved a number of its members to novel genera [9]. This proposal was based on phylogenetic analysis of 16S rRNA sequences and was later backed up by phylogeny of a selection of protein genes [10]. Currently, there are 71 recognized species belonging to the genus Clostridium sensu stricto [11] whose type strain is $C$. butyricum. The species $C$. difficile is no longer part of this genus, as it was placed in the novel genus Clostridioides, together with C. mangenotii $[9,12]$.

The main virulence determinants of $C$. difficile are toxin $\mathrm{A}$ (ToxA) and toxin $\mathrm{B}$ (ToxB), which are encoded on pathogenicity locus (PaLoc) by $t c d \mathrm{~A}$ and $t c d \mathrm{~B}$ genes, respectively, together with three regulatory genes $t c d \mathrm{C}$, $t c d \mathrm{E}$, and $t c d \mathrm{R}$ [13]. It has been suggested that PaLoc is a mobile element [14], in which case transfer of the complete locus can convert a non-toxigenic strain into a toxigenic one [15]. In addition, a third toxin may be present, known as binary toxin or CDT (short for $C$. difficile toxin, not to be confused with the cytolethal distending toxin of Gram-negative bacteria). This toxin is encoded by $c d t \mathrm{~A}$ and $c d t \mathrm{~B}$ genes and belongs to the Iota-family of toxins to which also Clostridium perfringens toxin belongs [16], although the relevance of CDT in clinical disease is still discussed [17].

Genetic differentiation of $C$. difficile strains is important to identify possible nosocomial outbreaks, in which multiple patients are infected by a single strain. A common method for genetic differentiation is multilocus sequence typing (MLST). By this method, $C$. difficile strains have been classified into six phylogenetically different clades (clades 1 to 5 and C1) [13], although Clade C1 is not defined in the MLST database collected at the University of Oxford, UK. These clades may contain both pathogenic and non-pathogenic strains, but the vast majority of strains produce one or more toxins [13]. This suggests that MLST may not be an ideal approach to highlight differences among the toxin gene repertoire of the isolates under study.

Technical developments now allow routinely sequencing whole genome sequences (WGS), instead of a limited number of gene fragments only. A large number of WGS from $C$. difficile strains are already available in the public domain and these sequences have been useful in multiple ways. WGS characterization has been helpful in the study of $C$. difficile infections (CDI) [18], and it identified the true genetic diversity that exists among C. difficile isolates, which was originally assessed by identification of single-nucleotide variants (SNVs) within a limited set of known genes only [5]. In addition, WGS has assisted epidemiological investigations, as it is superior in identifying CDI transmission sources, in particular in patients with recurrent CDI infections [19-21]. By means of WGS, it was recently shown that most cases of CDI in hospitalized patients are due to endogenous strains carried by the patients who were asymptomatic prior to their hospitalization, while in the hospital, exposure to high doses of antibiotics may favor the growth of the bacteria, resulting in symptomatic CDI [22]. In such a scenario, even non-toxigenic strains can pose a risk, since they can acquire the PaLoc by horizontal gene transfer, carry alternative virulence genes, or result in the condition of dysbiosis $[15,23]$. WGS has further been applied to assess the effectiveness of fecal transplants in severe CDI cases [7]. Lastly, metagenomics, in which all DNA present in a (clinical) sample is being assessed, is increasingly being applied to fecal analysis and may become a commonly applied technique in medical microbiology in the near future. Its main advantage is the identification of microorganisms without the need of culture, saving time in an outbreak investigation [24].

WGS results in a lot more data than what is assessed by SNP-based analysis or MLST. Since complete proteomes can be predicted, these can be subjected to average aminoacid identity (AAI) analysis, a method that compares all conserved protein-coding genes present in a given set of genomes, clustering strains into groups that sharing more than $95 \%$ AAI $[25,26]$. This method has proven to have higher resolution power at the species level than comparison of $16 \mathrm{~S}$ rRNA or MLST, since it assesses a far larger fraction of the genome [24].

Here, we compared all available $C$. difficile genomes by AAI analysis based on WGS data, starting with a comparison of taxonomic type strains of Firmicutes. The aims were to (1) determine if AAI analysis can confirm the status of $C$. difficile as a unique species; (2) assess if AAI produces groupings within the species that are of clinical relevance, to accurately identify 
pathogenic strains. For this second aim, the findings were compared to presence or absence of the toxin genes in different strains. As aim (3), we investigated whether metagenomics can identify CDI together with its associated strain (s), through a detailed analysis of published whole shotgun metagenomic sequences.

\section{Methodology}

\section{Average Amino Acid Identity Analysis}

AAI analysis was performed as previously described [27] and briefly summarized here. Amino acid sequences of all proteins from the analyzed genomes were extracted from their original GenBank accessions. AAI analysis was then carried out for every possible pair of genomes. The conserved reciprocal best match for each protein from each genome pair was first identified using UBLAST [28] with a cutoff of $30 \%$ sequence identity and a required minimum of $70 \%$ alignment length of the query sequence. For each pair of genomes, the average amino acid identity was then calculated based on the identities of all conserved reciprocal best matches, a calculation that is not always symmetrical. In such cases, the average of the two AAI values was assigned to each pair of genomes. Genomic clusters were then generated from the AAI values with a default cutoff of $95 \%$ (unless stated otherwise), meaning that members from different clusters cannot share more than 95\% AAI identity, and members within a cluster have paths consisting of edges connecting isolates with AAI $>95 \%$. The exact way how this clustering was done is described elsewhere [25, 26].

The first AAI tree was produced of all 25 taxonomically valid type strains of Firmicutes for which whole-genome sequences were available at the time of analysis. The list of type strains was obtained from the Names4Life website (www. namesforlife.com, accessed on 14 March 2017). The pairwise comparisons used to calculate AAI values for this set of genomes involved between 236 and 1766 genes (666 genes on average). The AAI tree was built with BIONJ [29] to dissimilarities of AAI values (100\% minus AAI).

For comparison of all Clostridia members, the GenBank database was accessed on February 12, 2017, and all available complete genomes and chromosomes $(n=234)$ within the Clostridia class were downloaded. This selection was restricted to completely sequenced genomes and included 8 genomes from $C$. difficile. The AAI values of the Clostridia selection involved compared between 131 and 5017 genes (955 genes on average).

A third AAI tree was built using all $663 C$. difficile genomes that had genome quality scores $>0.8$ as defined elsewhere [30], this time including complete genomes as well as draft genome sequences available at the time of analysis. The dataset contained 653 sequences described as obtained from $C$. difficile, completed with 10 genomes with no species designation but presumed to be $C$. difficile based on gANI (genome-wide average nucleotide identity) [31]. Two genomes of Clostridioides mangenotii (GCA 000498755 and GCA 000687955) were added to serve as an outgroup. A tree that contains over 600 branches would be hard to read, and since many genomes are extremely similar, the tree would end in many very short branches. For graphical representation of such a large dataset, branches that contained identical or highly similar members were collapsed. We attempted to define a suitable cutoff for such a collapse by varying the required percentage of similarity within this dataset as described in the results. Bootstrap values were calculated conceptually similar to alignment-based trees: among the reciprocal conserved protein pairs identified for a given pair of genomes, we selected pairs randomly with replacement of as many as the number of the original pairs and repeated this procedure 100 times, resulting in 100 bootstrap AAI values for that pair of genomes. Then, 100 bootstrap AAI trees were generated and bootstrap values were calculated, defined as the occurrence of clades of the AAI tree in 100 bootstrap trees.

\section{In Silico MLST}

The seven gene fragments of housekeeping genes $a d k$, atpA, $d x r, g l y A, \operatorname{rec} A, \operatorname{sod} A$, and $t p i$ were extracted from the $C$. difficile genomes by NBLAST using the sequence of allelle number 1 as the query; these query sequences were extracted from the MLST database (https://pubmlst. org/cperfringens/) collected by the University of Oxford. The best NBLAST hit with each genome was retrieved, sequences were concatenated, and a NJ tree was constructed by Muscle [32]. Redundancy was removed by deleting multiple sequences per sequence type (ST), recording the number of members per ST. For comparison, the complete genes instead of MLST fragments were also concatenated and analyzed.

\section{Identification of Toxin Genes by PFAM Domain Searches}

Prodigal software was used to identify all protein-coding genes across all analyzed $C$. difficile genomes [33]. The Pfam domains in the proteins of these genomes were identified using HMMER 3.1b2 [34] to scan across the 16,306 profile hidden Markov Models in the Pfam database version 30.0 [35]. Presence of genes coding for toxin A or toxin $\mathrm{B}$ was identified on the basis of presence of the Pfam domains PF12918, PF12919, PF11713, and 
PF12920. CDT protein A was identified on the basis of presence of the Pfam domain PF03496 and CDT protein B by presence of PF07691 and PF03495.

\section{Read Coverage Analysis from Metagenomic Data}

To assess if metagenomics can be used for identification of CDI, we investigated the recently published shotgun metagenomes produced from stool samples of patients with suspected or confirmed CDI in two different hospitals located in Canada [6] and Italy [33]. A total of 228 metagenomic samples were available from the Canadian study, including CDI cases and controls. We blinded our analysis, not knowing which of these samples were from controls and which were from patients. Of the 15 Italian metagenomic samples available, we only analyzed two datasets from clinically confirmed CDI cases, as diagnosed by the authors [36]. Reads were downloaded from NCBI BioProjects PRJNA297252 and PRJNA297269 at NCBI's Sequence Read Archive (SRA) database. The SRA metagenomic data were converted into FASTQ format using 'SRA Toolkit' software and then aligned to the 663 C. difficile genomes using Burrows-Wheeler analysis (BWA) software version 0.715 [37] using default parameters. All reads were compared to $C$. difficile sequences and the $C$. difficile genome to which the most abundant reads were matched was then identified. The genome coverage of the metagenomic reads against that reference genome was determined by using the genomecov function in BEDTools software [38]. For four datasets that had a genome coverage of over $50 \%$, the reads were plotted using the Integrative Genomics Viewer (IGV) [39] to illustrate the presence or absence of toxin operon genes, using the complete genome of $C$. difficile NC_009089.1 as a template. A sample with lower genome coverage was included as a control. The five sequences presented here include SRR2565933, SRR2565934, and SRR2565548 (BioProject ID PRJNA297252) and SRR2582247 and SRR2582248 (BioProject ID PRJNA297269).

\section{Results and Discussion}

\section{Average Amino Acid Analysis of Taxonomic Type Strains Belonging to Firmicutes}

A cladogram tree was generated based on AAI analysis using 25 genomes from Firmicutes taxonomic type strains (Fig. 1). All genomes cluster in their expected class, although the Clostridia class (shown in brown) is split up into two large clusters. The first Clostridia cluster starts with $C$. difficile and for the second member of this genus, C. butyricum, its closest neighbor is
Tissierella praeacuta, which at first seems to be strange. However, the type strain of this species and that of Clostridium hastiforme have been shown to be identical, so that the two genus names can be considered synonyms, with priority for T. praeacuta [40]. The first Clostridia cluster is followed by a cluster of Negativicutes, represented by type strains of the genera Veillonella, Selenomonas, and Acidaminococcus (shaded blue in the figure). These Firmicutes produce negative Gram stains and, like all Gram-negatives, contain two cell membranes. Nevertheless, based on their genome content, they are distant to both wellcharacterized Gram negatives and Gram positives, but slightly closer to Gram-positives [41]. All Bacilli type strain representatives compared in this analysis are found together in the next cluster, indicated in green. The only exception to the expected position in the tree is the type strain of Symbiobacterium thermophilum, which is found as part of the Bacilli cluster although this genus is considered to belong to the Clostridia, as indicated by the brown shading in the figure. From its position in the tree, it is obvious that the type strain of Symbiobacterium thermophilum shares considerable characteristics with Bacilli. That is no surprise, as these organisms live in strict symbiosis with Bacilli, which has probably resulted in frequent gene swaps [42]. From Fig. 1, we conclude that AAI analysis is able to correctly group Firmicutes genomes according to recognized taxonomic groupings.

\section{Average Amino Acid Analysis of Completely Sequenced Clostridia Members}

The next AAI analysis concentrated on completely sequenced genomes of the Clostridia only. A total of 234 genomes were compared, this time not representing a selection of type strains but all genomes that were completely sequenced at the time of analysis. In Fig. 2, distinct clusters are colored, and some of these nicely overlap with species, for instance the clusters of Moorella thermoacetica, Caldicellulosiruptor saccharolyticus, or the eight fully sequenced $C$. difficile genomes that were included, highlighted in bright blue. The type strain of C. difficile (ATCC 9689/DSM 1296) that was used in Fig. 1 is not included here, as its genome sequence is not yet complete. Compared with the diversity observed in $C$. botulinum, the eight $C$. difficile genomes are much more similar to each other and are clearly separated from the other species included here. The closest neighbor of $C$. difficile on this tree is Paeniclostridium sordellii. Their close relationship was also demonstrated with a $16 \mathrm{~S}$ rRNA neighbor-joining phylogenetic tree [9]. Nevertheless, most clusters in Fig. 2 contain a mix of genera. This observation demonstrates that the taxonomic divisions within the Clostridia are still not well resolved. One 
Fig. 1 Amino acid identity (AAI) tree of 25 Firmicutes type strains. Members of the Clostridia are colored brown, Bacilli green, and Negativicutes blue

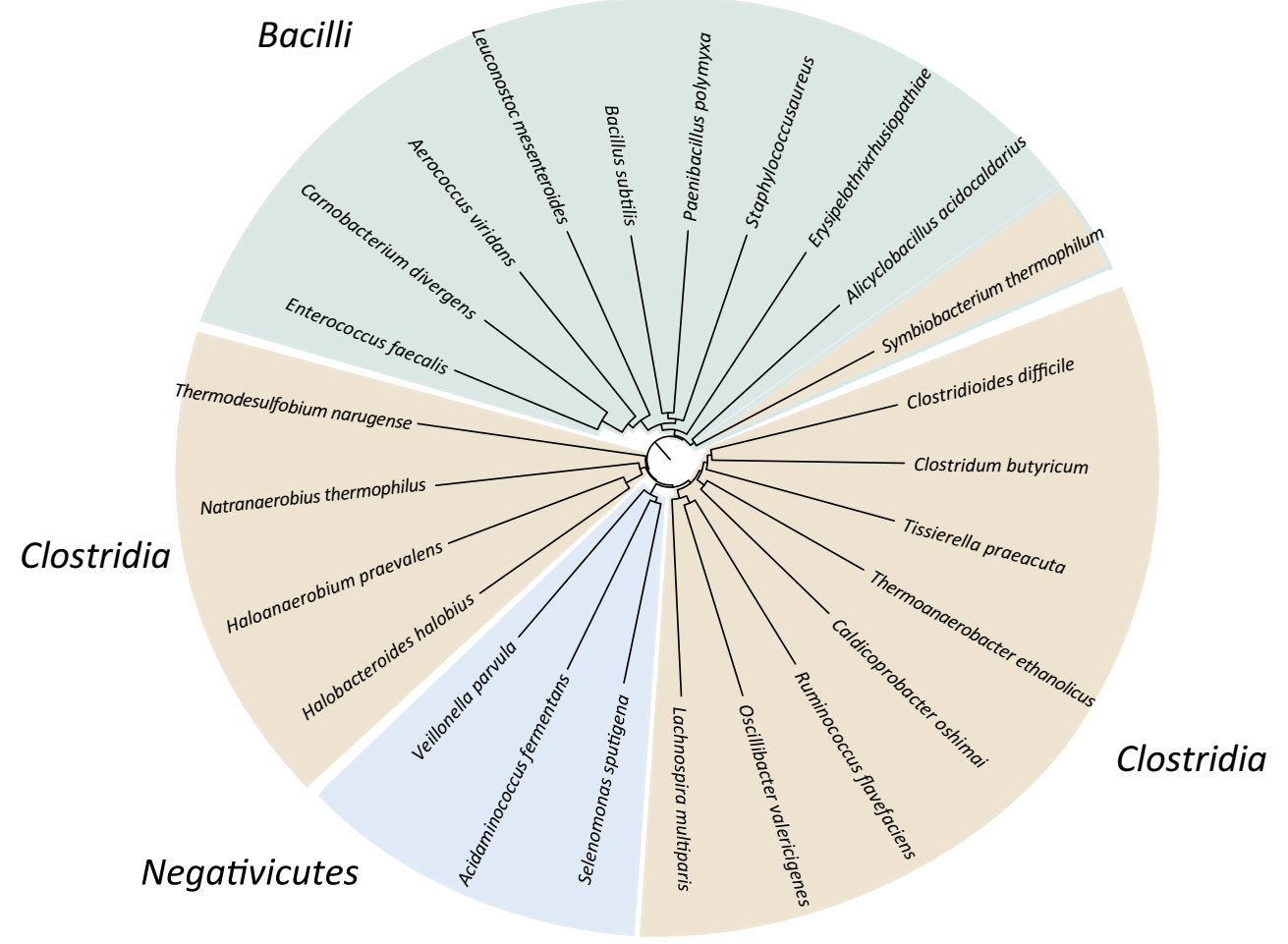

reason for this may be that the phylogenetic relationship on which taxonomy is partly based is using a relatively small selection of housekeeping genes only (such as gyrA or recA) as well as $16 \mathrm{~S}$ rRNA gene sequences. This approach works well for a number of bacterial families, where species are so clearly divided that these marker genes can reliably be used to define taxonomic divisions. In such cases, the findings obtained with analytic tools that assess the similarity of a large number of genes fit the taxonomic frame quite nicely. The AAI analysis applied here is such a multiple-gene comparison tool, and with this method, we have obtained results in good agreement with taxonomy for Pseudomonas species [27]. However, a number of Clostridia members presented in Fig. 2 are not completely resolved according to their taxonomic description, indicating that the currently used division is not always in accordance to their degree of genomic similarity.

\section{Average Amino Acid Analysis of 663 C. difficile Genomes}

Average nucleotide analysis (ANI) is often applied to bacterial taxonomy, whereby a cutoff of $94 \%$ is considered suitable [43, 44]. If amino acid-based AAI were to be added as an extra tool to compare strains within a species, the most suitable cutoff needs to be determined. We therefore analyzed the 663 currently available $C$. difficile sequences (complete genomes combined with those available as multiple contigs) by AAI. Ten genomes assigned to 'Clostridium sp. only' were included, and two
C. mangenotii genomes were added for comparison as this is the closest neighbor of $C$. difficile. Figure 3 shows a compilation of trees produced with different cutoffs from the $663 C$. difficile. At the default cutoff level that allowed $\geq 95 \%$ identity per branch, five branches were produced containing $C$. difficile genomes, two with single genomes and three representing clusters. The vast majority (640) of $C$. difficile genomes clustered together, as the default cutoff level could not distinguish these. This suggests that the vast majority of sequenced $C$. difficile genomes are relatively similar, but it also identifies 23 genomes that are more distant. This is relevant in view of the present trend to rely on in silico analysis for taxonomic assignment. The single genome branches in Fig. 3a represent one isolate for which no metadata were provided, and an isolate that had originated from a French study on $C$. difficile infections [45]. The four genomes that formed a separate cluster (colored blue in the figure) are from diverse geographic locations (two from Northern Iraq and two from the French national strain collection [13]). Likewise, the cluster comprising 17 genomes (shown in green) contained 9 isolates from a large Canadian initiative that sequenced a total of 470 strains, mixed with human isolates from Austria, Ireland, and Italy, and an equine isolate from Slovenia [46]), 2 samples supplied by a veterinary institute in Arizona for comparison in the Human Microbiome Project [47] and a 2007 isolate from a Food Safety Centre in Ireland [48]. The last genome in this cluster is from a strain described as a "non- 


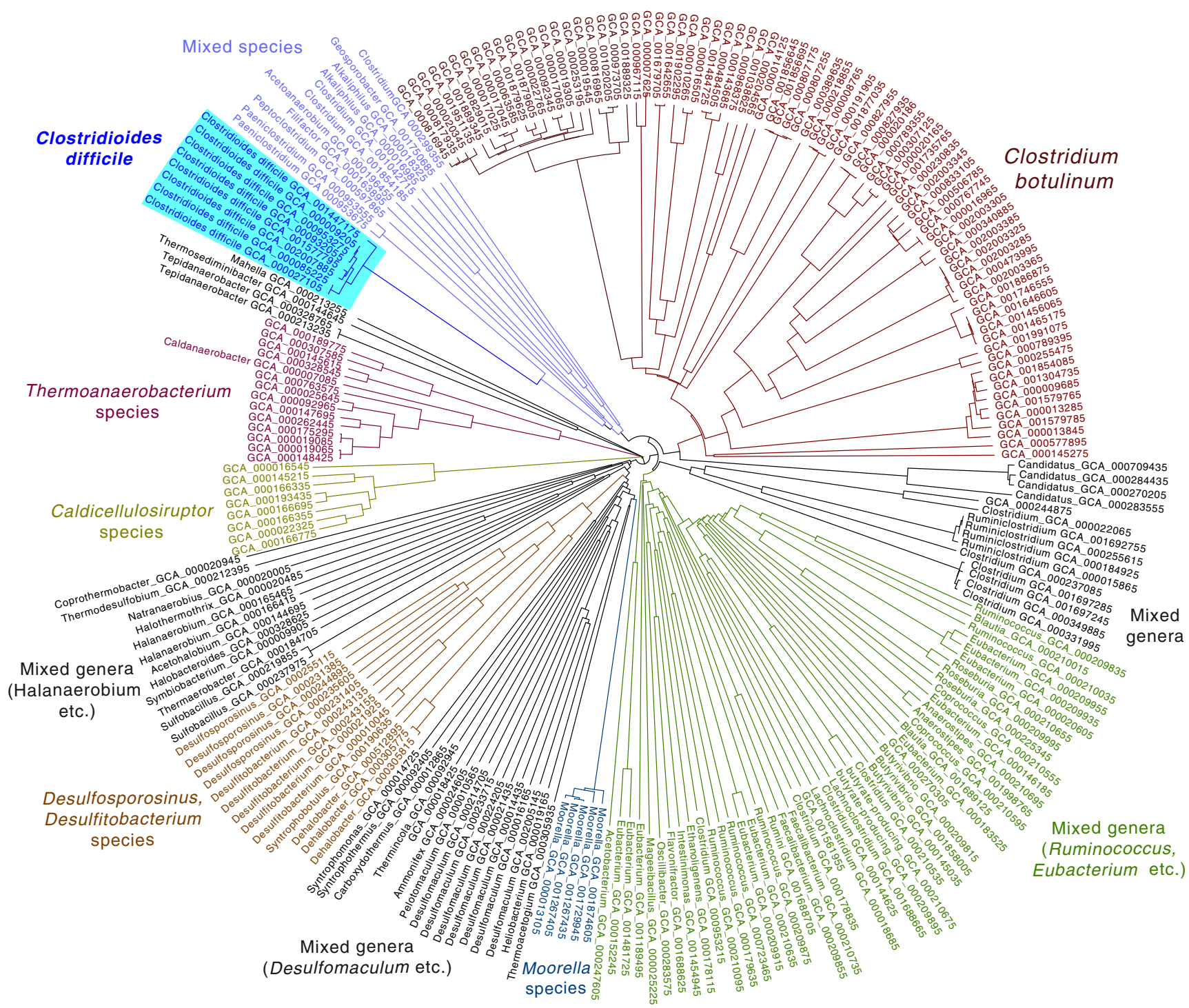

Fig. 2 AAI tree of 234 completely sequenced genomes belonging to the Clostrida class. The light blue shading (top left) identifies the 8 included Clostridioides difficile genomes. For clarity, branches are colored according to their main clusters with same-color descriptions added.
Branch labels only giving CGA numbers refer to genomes from strains described as 'Clostridium sp.', unless indicated otherwise with speciesspecific descriptions outside the tree epidemic human isolate', presumably from the USA. At least one of the strains in this 17 -member cluster was associated with severe diarrhea but several were described as non-pathogenic. The fact that this cluster contains strains from different continents and different host species suggests that the $95 \%$ cutoff of AAI groups allotropic strains with heterologous properties. Based on the available metadata, there is clearly a mix of virulent and nonvirulent strains.

By increasing the cutoff to $96 \%$ (panel 3B), the number of branches increases to 20 , of which 18 are clusters. The 17 strains that clustered together at $95 \%$ are maintained (now in cluster number 17), as is the cluster of four genomes (now cluster number 18). The remaining
640 genomes are now divided over 15 clusters, the largest one containing 168 genomes and the smallest 3 . The genomes that had been derived from 'Clostridium sp.' were distributed over different clusters, so these did not form a group of their own. This trend is continued when the cutoff is increased to $97 \%$, which maintains the clusters with 17 and 4 genomes (cluster numbers 23 and 24). However, at a level of $98 \%$, only the 4-genome cluster remains intact, while the 17 strains are now divided over 4 small clusters and 4 single-genome branches, as shown in panel $\mathrm{d}$ of Fig. 3. The small clusters are only partly region-specific, for instance, three Canadian strains are combined (cluster number 32), but another cluster combines the two Irish with the Italian and an Austrian strain 
A $95 \%$

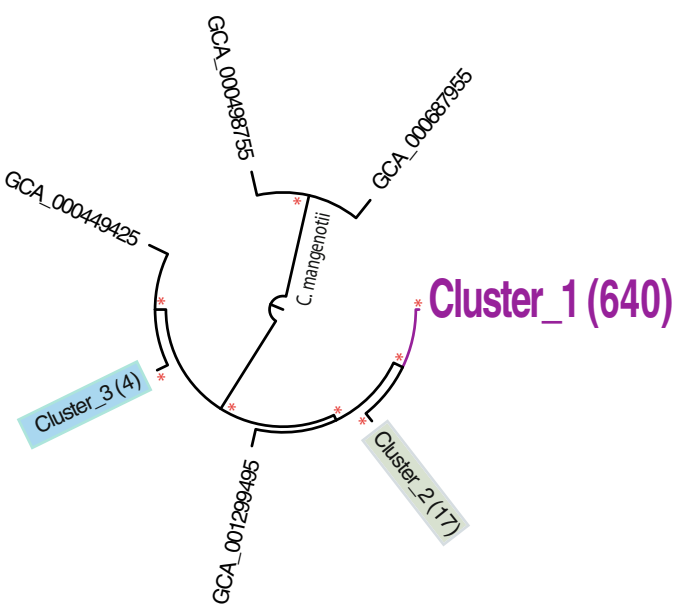

C 97\%

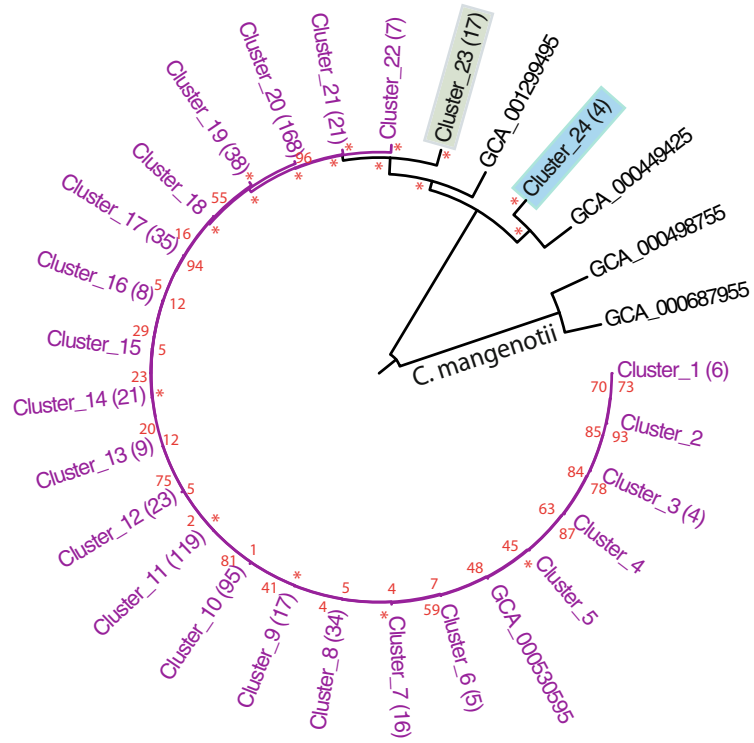

Fig. 3 AAI trees of 663 C. difficile genomes collapsed at a range of cutoffs from $95 \%$ (a) to $98 \%$ (d). The number of members of a cluster is indicated between brackets (only shown for clusters containing 4 or more members, not shown in d). Two clusters that are discussed in the

(cluster number 30). Continuing this analysis with a cutoff of $99 \%$ results in 92 clusters and 151 single-genome branches (results not shown).

From this analysis, we conclude that the included Clostridium sp. genomes can be considered to have been obtained from $C$. difficile members. Twenty-three submitted $C$. difficile genomes are clear outliers, which form two clusters and two single-genome branches. Further, with an increase of the chosen cutoff, larger clusters break up into smaller ones, and this is a continuous process.
B 96\%
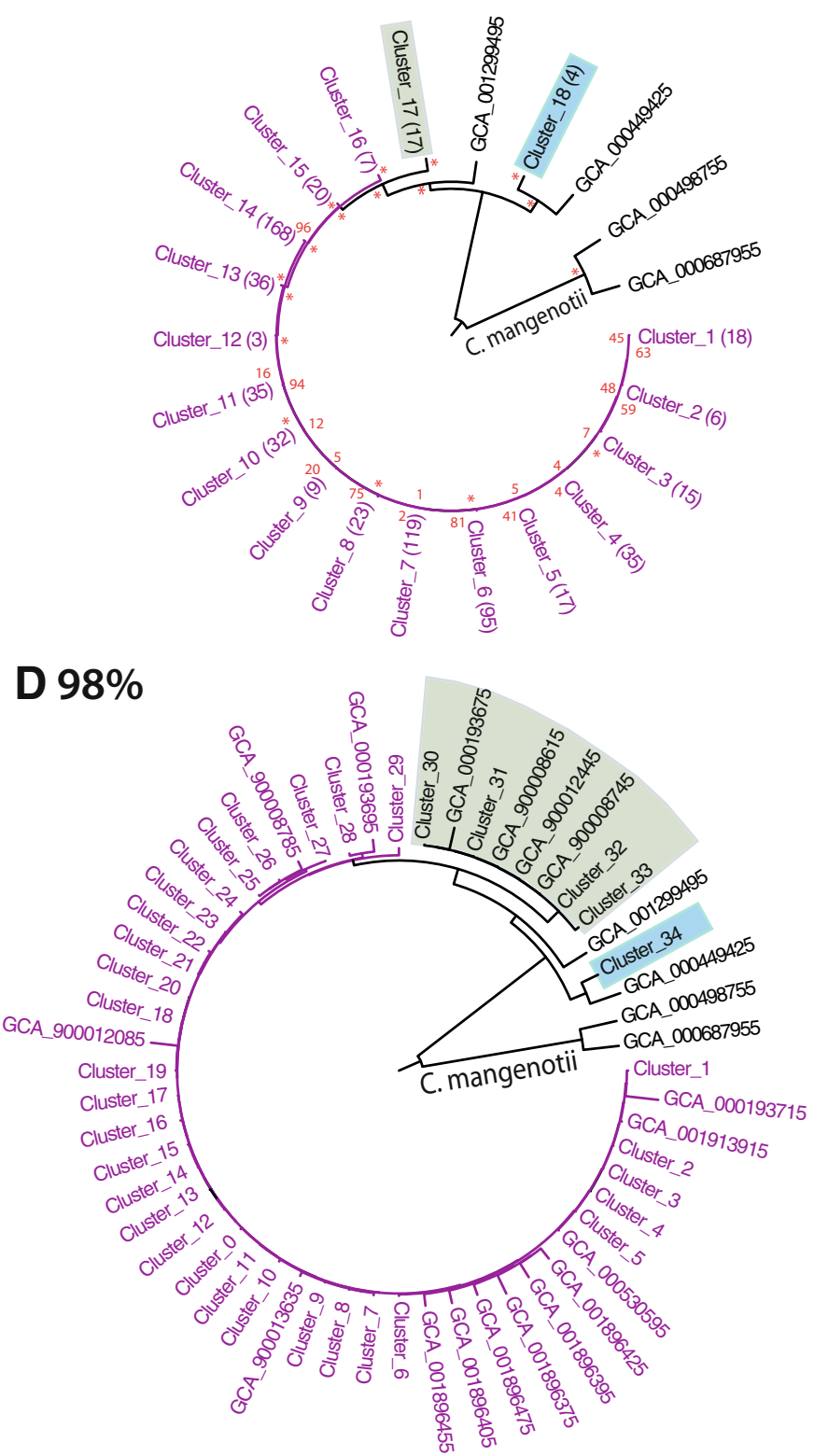

text are marked in blue and green. Numbers in red (not shown in d) give the confidence of nodes and clusters, with an asterisk for value 100. An unscaled version with all confidence values added is available as supplementary Fig. S1

\section{MLST Analysis of $C$. difficile Genomes}

We also analyzed the same set of $C$. difficile genomes by MLST, which is often applied to compare strains within a species [49, 50]. Complete allele fragments could be extracted from only 607 sequences. A non-redundant tree of the concatenated gene fragments is shown in Fig. 4. A table with genome accession numbers and their corresponding MLST sequence type and clade is included as Supplementary Table S1. Most of the genomes belong to MLST clades $1(n=339)$ and $2(n=161)$. The single 
Fig. 4 In silico MLST of 607 C. difficile genomes. a A nonredundant $\mathrm{NJ}$ tree with bootstrap values in red, where sequence types (ST) are given with the number of members in brackets. The arrow points to the ST01 branch with 146 members. ST00 indicates that a sequence type is not assigned to that allele combination. The four MLST clades are indicated and four genomes shaded in blue are the same that formed the fourmember cluster in Fig. 3. b Part of an NJ tree based on complete MLST genes coding sequences. The red line separates clade 1 and clade $2 \mathrm{STs}$. A number of clade 1 STs are more similar to clade 2 sequences (thus colored green, left of the red line), and ST2 is split between the two clusters
A

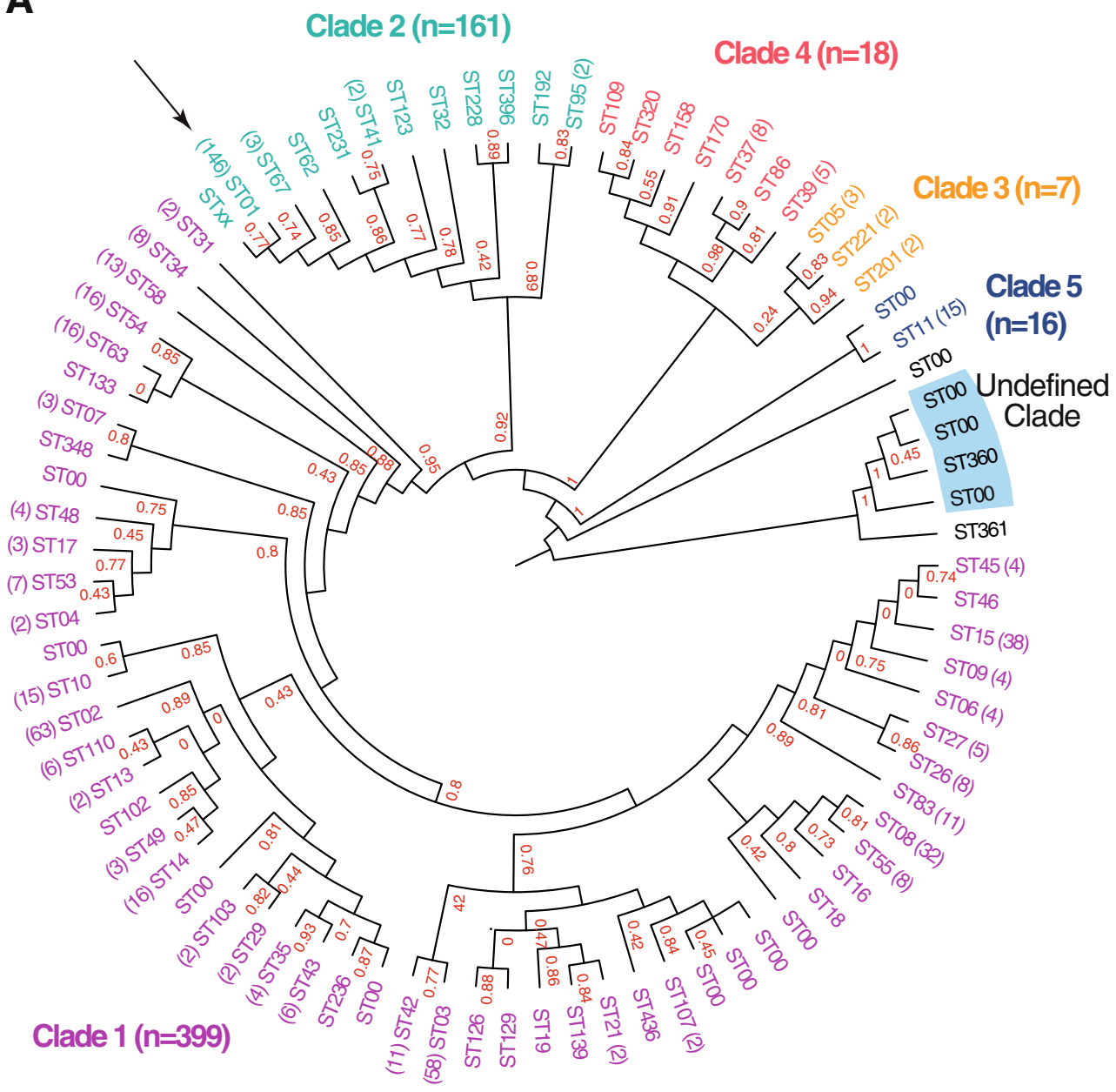

B

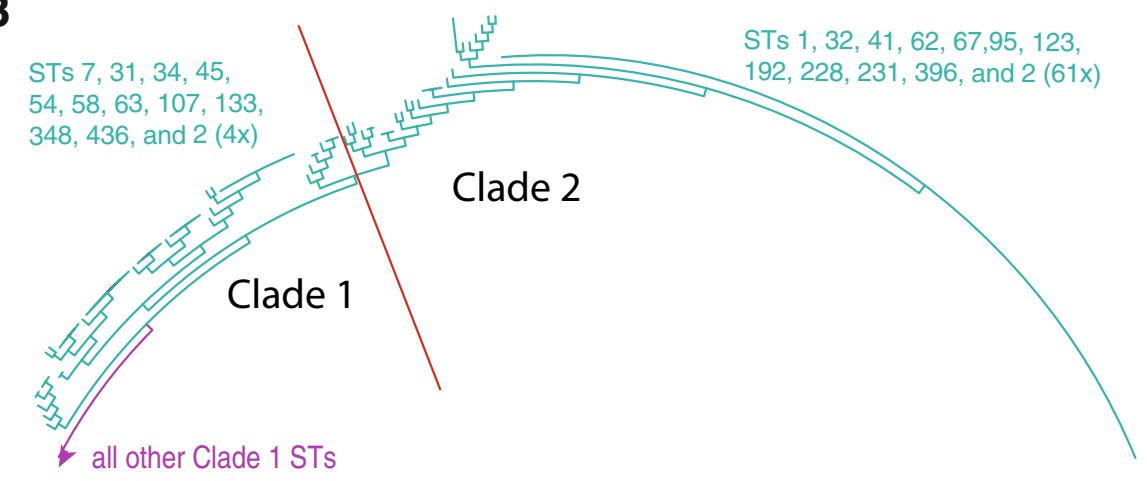

most frequently represented sequence type is ST01 (part of clade 1) which was found for 146 genomes. Clades 3 and 4 have 7 and 18 representatives, respectively. The AAI cluster with 17 members belongs to clade 5 (for one the MLST type could not be determined). One of the two genomes that formed an outlier by AAI analysis had ST361 that grouped with four genomes in an unassigned clade; these were the four members that by AAI remained in one cluster even at $98 \%$ (Fig. 3d). The other
AAI outlier was of an unassigned ST positioned between clade 5 and the unassigned clade. Supplementary Table S1 compares the STs with AAI cluster numbers at 98 and $97 \%$. In most cases, genomes with identical ST cluster together by AAI but there are exceptions. For instance, ST1 (clade 2) is very homogeneous, but three ST1 members are placed at a large distance from their peers in the AAI trees. ST54 members (clade 1) are divided over 5 AAI clusters and include 3 single 
branches by that analysis, indicating that this ST is relatively heterogeneous.

Although clearly separated by MLST, the clades 1 and 2 were not clearly visible in the AAI trees of Fig. 3. We therefore re-analyzed the seven MLST genes, this time including complete coding sequences instead of the typically used MLST fragments. The result (Fig. 4b) showed that clades 1 and 2 are distinct but closely related, which explains why this division was not visible by AAI. In fact, a number of STs that are part of clade 1 are closer related to clade 2 members, based on their full-length MLST gene assessment, and four ST2 members are mixed with clade 1 STs (these 71 genomes are identified in Table S1). This illustrates that the distinction in clades based on MLST fragments is somewhat arbitrary and depends on how these fragments were chosen. Thus, the clades do not necessarily represent truly distant lineages.

\section{Presence of Toxin Genes in C. difficile Genomes}

Next, we assessed the presence of the $C$. difficile toxin genes, by searching for their typical Pfam domain architecture. Out of 663 genomes, 535 contained the Pfam domains indicative of presence of toxin $\mathrm{A}$, toxin $\mathrm{B}$, or both. This means that approximately $81 \%$ of the sequenced $C$. difficile genomes were potentially toxigenic. The two toxins cannot be distinguished based on their Pfam domains, but when two domains are found per genome, this indicates presence of two genes, presumably $t c d \mathrm{~A}$ and $t c d \mathrm{~B}$. This was the case in 483 or $73 \%$ of all genomes analyzed. Most of the clusters produced at various degrees of collapse combined toxigenic strains with those lacking the telltale toxin Pfam domains. In Fig. 5, the results are presented at 98\% clustering for a graphical representation. This suggests that the toxins can be present or absent independent of genetic background, which is supportive of their presumed mobility $[14,15]$. Of note is that some clusters (e.g., cluster numbers 23 , 24 , and 25 at $98 \%$ containing 6,3 , and 38 strains, respectively) were homogeneous for lacking ToxA and ToxB, which is a striking finding giving the high incidence of these genes. Only a few of these isolates were epidemiologically related, as exemplified by the 38 strains in cluster 25 , of which 20 belonged to the large strain collection from Quebec that was sequenced, but it also contained two environmental isolates from the UK, four related strains from Japan, three strains from the Pasteur Institute collection, one from Australia, one from Northern Iraq, and 7 that were sequenced by the University of Maryland. This would suggest that the cluster represents a group of globally distributed non-toxigenic isolates, though their genomes are not completely identical. At $99 \%$ collapse, these genomes are redistributed over three clusters (results not shown) though again these do not completely separate according to geographic origin. Again, these findings would suggest

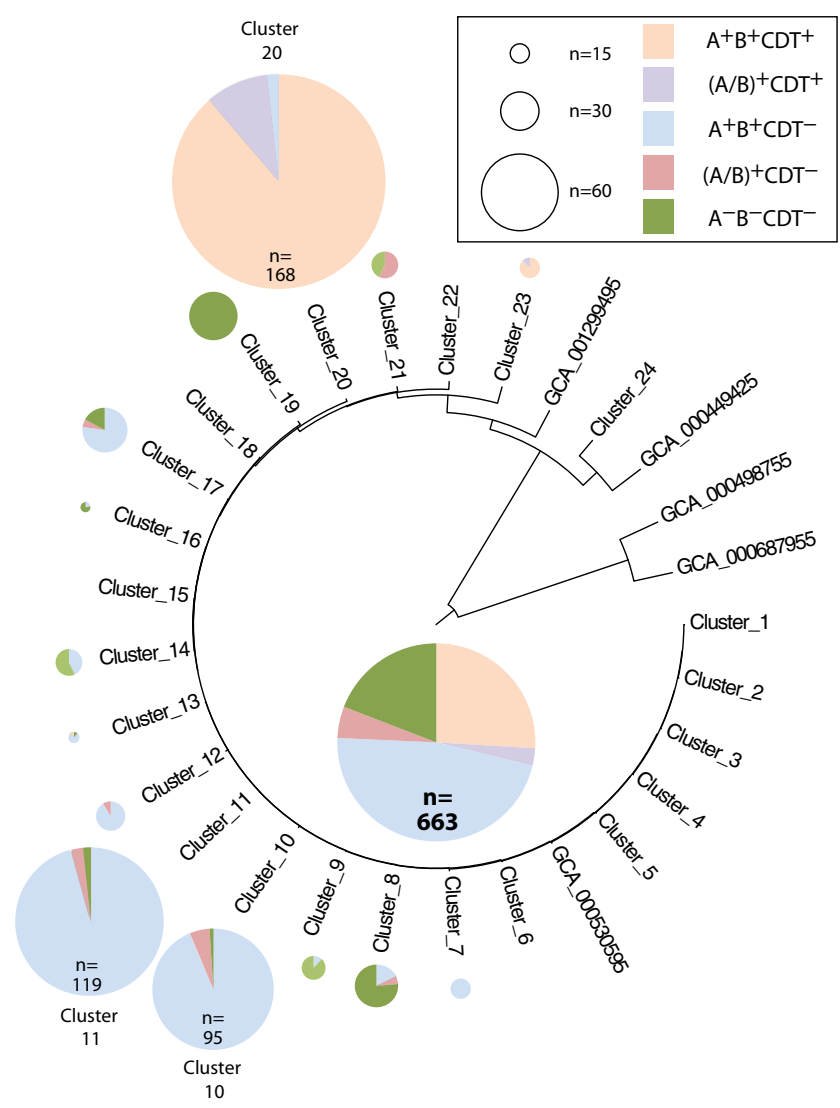

Fig. 5 Distribution of toxin genes in the clusters of $C$. difficile collapsed at $97 \%$. Pie charts are only shown for clusters containing 9 members or more, and those shown outside the radial tree are scaled. The distribution in all 663 genomes combined is shown inside the radial tree

that a collapse at $98 \%$ is sufficient to identify closely related genomes of $C$. difficile, acknowledging that variation within the generated clusters exists, and strains from various geographic locations may be combined that share a high degree of protein content similarity.

We also searched for presence of Pfam domains indicative of the two proteins that make up CDT. Only 191 genomes contained (after translation) the Pfam domains typically present in CdtA, and two more contained CdtB domains. Interestingly, nearly all of the CDT-positive genomes also contained one or two copies of ToxA/B: 172 genomes contained ToxAB (deduced from presence of two copies of the toxin-specific Pfam domains) and 18 contained one copy. Since all genomes lacking ToxA/B also lacked CDT (with one exception), it seems the presence of these different toxins is highly overlapping. Only one genome analyzed here contained CDT but not ToxA/B (A-B - CDT+), while 310 genomes reported $\mathrm{A}+\mathrm{B}+\mathrm{CDT}-$; the latter is most often found in the investigated genome collection. The presence of CDT in strains lacking ToxA/B has been described before [46-51], but apparently, this is rather uncommon, as we find this in only $0.15 \%$ of the strains for which a genome sequence is currently available. 


\section{Metagenomic Analysis Can Identify C. difficile Infection in Stool Samples}

Lastly, we analyzed metagenomic data obtained from two studies involving CDI patients. A blinded set of 228 metagenomic datasets obtained from fecal samples from Canadian CDI patients and controls [4] was screened for presence of $C$. difficile sequences. Two metagenomic samples from an Italian study [37] were also analyzed. The analysis identified two Canadian samples, $\mathrm{C} 1$ and $\mathrm{C} 2$, that produced strong coverage of $C$. difficile sequences. All other metagenomic samples from that source resulted in very poor coverage, of which $\mathrm{C} 3$ is presented here as it retrieved the highest number of reads of the 226 samples. The reads from the two clinically confirmed Italian cases also contained $C$. difficile sequences. Of the five samples presented here, between 0.25 and $9.05 \%$ of the total reads mapped to a $C$. difficile reference genome (Table 1). The abundance of a species in a metagenomic sample can be roughly estimated from the read coverage, so this observation indicates that $C$. difficile was present at low levels in the gut of four of these individuals. Only the stool of patient I1 seemed to have high numbers of $C$. difficile as deduced from the read coverage (Table 1). Those reads that could be mapped to a $C$. difficile genome covered between 9.69 and $98.76 \%$ of that genome. Thus, in the case of patients $\mathrm{C} 1$ and $\mathrm{C} 2$, nearly a complete $C$. difficile genome was present in the metagenomic reads. The data from the Italian study, which had resulted in approximately 10 times fewer total reads (Table 1), still covered around $52 \%$ of a $C$. difficile genome. In contrast, the reads obtained from feces of patient $\mathrm{C} 3$ only covered $<10 \%$ of the best matching $C$. difficile genome, although there were over 139,000 reads mapped to that species in total. The vast majority of those reads mapped to a few short specific region of a $C$. difficile genome only, which happened to be fragments of $16 \mathrm{~S}$ and $23 \mathrm{~S}$ rDNA and one tRNA_met gene (results not shown). Of note is that these RNA genes are present in multiple copies (between 13 and 15 copies of the ribosomal gene locus and about 8 copies of the tRNA_met gene are typically present in a $C$. difficile genome) which may explain why these sequences were picked up. These results suggest that the stool of patient C3 contained very low levels of $C$. difficile, whose presence could only be detected based on multiple gene copies of particular RNA genes. In contrast, the much better coverage of half or a nearly complete $C$. difficile genome in samples $\mathrm{C} 1, \mathrm{C} 2, \mathrm{I} 1$, and I2 suggests that these contained higher numbers of this pathogen.

For the metagenomic sequences obtained from Canadian patient $\mathrm{C} 1$, the reads that matched $C$. difficile produced the best match with the genome of $C$. difficile strain 5.3, isolated in Australia [52]. For patient $\mathrm{C} 2$, the best match was

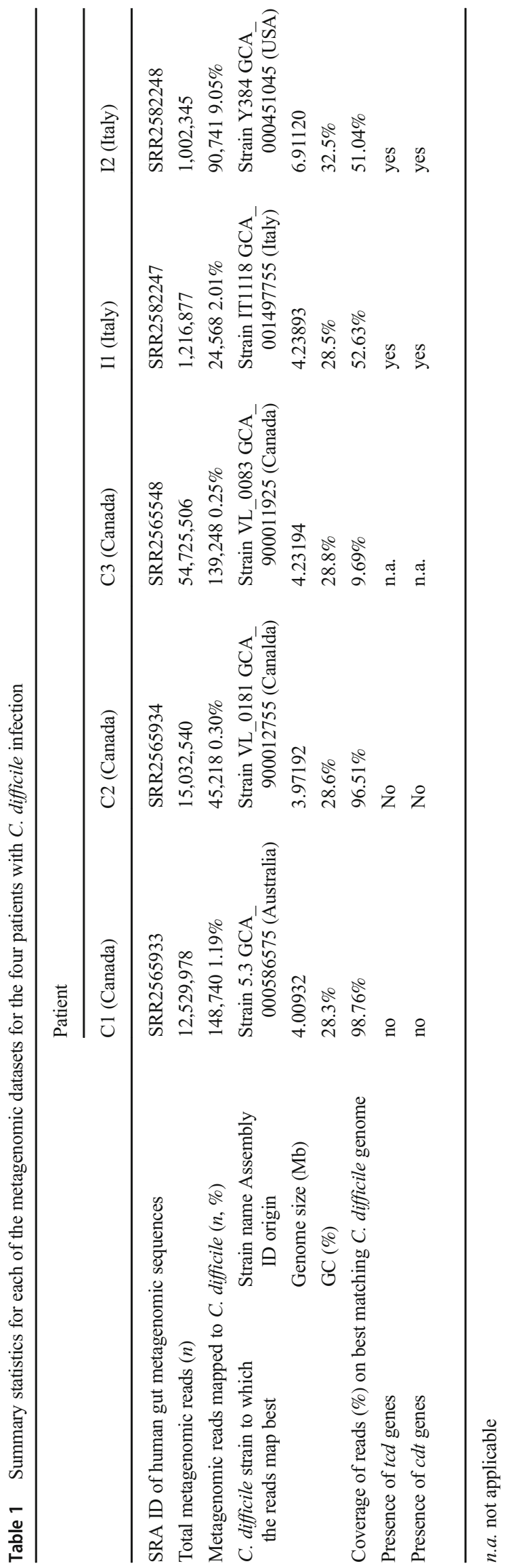




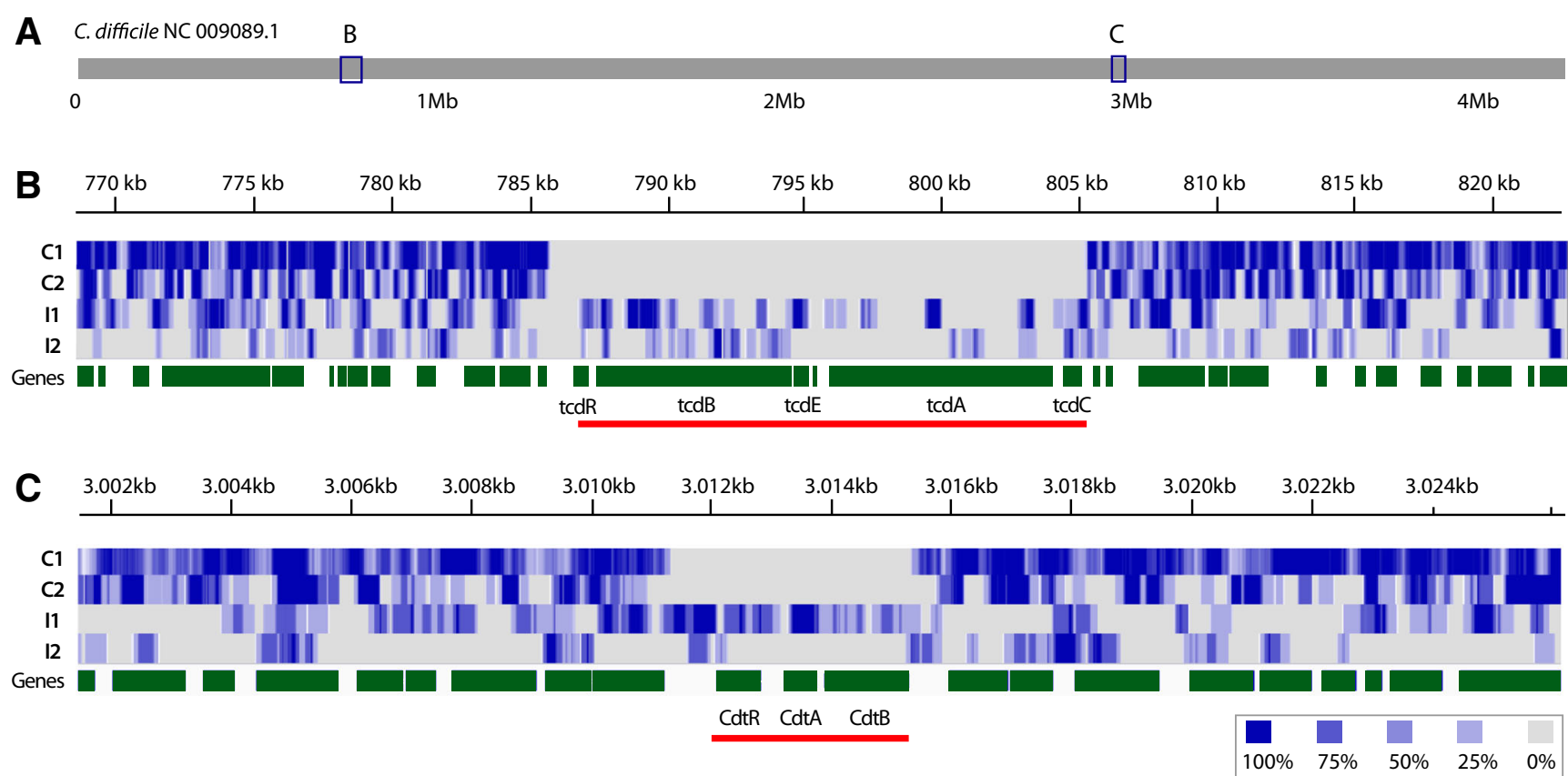

Fig. 6 Genome coverage for the four toxin operons within $C$. difficile reference genome NC 009089.1. a The position of locations shown in $\mathbf{b}$ and $\mathbf{c}$ on the genome of the reference genome. $\mathbf{b}$ The read coverage of the tcd locus (red underlined) and its direct environment for the four

obtained with strain VL_0181 which had been isolated from Canada. However, these two strains did not carry the $t d c$ or the $c d t$ operon, thus resulting in gaps in their sequence, as illustrated in Fig. 6. Indeed, $t d c A, t d c B$, and $c d t$ sequences were absent from the metagenomic reads of $\mathrm{C} 1$ and $\mathrm{C} 2$, demonstrating that for these patients, CDI was caused by a non-toxigenic strain. In addition, our analysis shows that the two patients had been infected by different strains. Similarly, different strains were identified in the two patients from Italy. Patient I1 resulted in sequences most closely matching to $C$. difficile strain IT1118 with ribotype 018, which was responsible for outbreaks occurring in Italy, South Korea, and Japan [53], while patient I2 possessed a strain whose reads were most similar to $C$. difficile strain Y384, isolated in a hospital of Pennsylvania, USA. In these two cases, the genomes contained $t c d$ and $c d t$ operons, which is illustrated in Fig. 6.

In total, these data show that metagenomic analysis of stool samples can identify presence of $C$. difficile, and the degree of genome coverage can be taken as a measure for abundance of the organism. In the future, as the cost of metagenomic sequencing becomes more affordable and faster, this approach might become economically feasible for more routine analysis. We further conclude that, although patients may be diagnosed with a CDI in the same hospital, their infection was unlikely due to a common nosocomially transferred strain. More likely, endogenous, community-acquired strains, may have been responsible for these analyzed cases. metagenomic samples with heatmap colors in gray/blue as indicated. Gene locations are shown in green. $\mathbf{c}$ The $c d t$ locus (red underlined) and its direct environment of the same metagenomic samples. Data taken from BioProjects PRJNA297252 and PRJNA297269

\section{Conclusions}

The cluster analysis presented here has shown that for different members of Firmicutes, AAI clustering provides valuable insights on similarities that broadly agree with taxonomic position. At the level of genera and species within Clostridia, the clusters are less well resolved, as various genera are mixed. The taxonomic classification of $C$. difficile has encountered difficulties in the past. The WGS analysis presented here was based on AAI, which captures a large fraction of protein gene content. That clearly identified all analyzed $663 \mathrm{C}$. difficile members belong to a single species that is distinct from its closest relatives. At the level of strains within the $C$. difficile species, AAI analysis groups the vast majority of genomes within one cluster at $95 \%$ cutoff. This cluster subdivides as the cutoff for similarity is increased, but a clear optimal cannot be identified. Most genomes with identical STs group in AAI clusters, but there are exceptions. MLST clade 1 contains a number of STs that are more similar to members of clade 2 . The analysis further showed that the toxin genes are unevenly distributed over the strains.

Metagenomic analysis of stool samples can identify cases of CDI, and CDI-causing strains can be atoxigenic. Detection of multi-copy RNA genes exclusively in metagenomic reads may be indicative of low numbers of $C$. difficile in stools. The detected sequences suggest CDI cases may be caused by different strains in patients form the same hospital. These findings support evidence for the acquisition of the pathogen within the community, with autogenous strains causing the infection. The onset of 
symptoms during hospitalization may be a result of treatment rather than in-hospital spread of an epidemic strain.

Acknowledgements We thank Dr. George Garrity for providing the selection of type strain genomes.

Funding This project was partly supported by the Translational Research Institute (TRI), grant UL1TR000039 through the NIH National Center for Research Resources and National Center for Advancing Translational Sciences; by NIH/NIGMS grant 1P20GM121293; and from the Helen G. Adams \& Arkansas Research Alliance Endowment in the Department of Biomedical Informatics, College of Medicine at UAMS. The content is solely the responsibility of the authors and does not necessarily represent the official views of the NIH.

\section{Compliance with Ethical Standards}

Conflict of Interests The authors declare that they have no conflict of interests.

Open Access This article is distributed under the terms of the Creative Commons Attribution 4.0 International License (http:// creativecommons.org/licenses/by/4.0/), which permits unrestricted use, distribution, and reproduction in any medium, provided you give appropriate credit to the original author(s) and the source, provide a link to the Creative Commons license, and indicate if changes were made.

\section{References}

1. Ofosu A (2016) Clostridium difficile infection: a review of current and emerging therapies. Ann. Gastroenterol 29:147-154. https:// doi.org/10.20524/aog.2016.0006

2. Peery AF, Dellon ES, Lund J, Crockett SD, McGowan CE, Bulsiewicz WJ, Gangarosa LM, Thiny MT, Stizenberg K, Morgan DR, Ringel Y, Kim HP, DiBonaventura MD, Carroll CF, Allen JK, Cook SF, Sandler RS, Kappelman MD, Shaheen NJ (2012) Burden of gastrointestinal disease in the United States: 2012 update. Gastroenterology 143:1179-1187.e1173. https://doi. org/10.1053/j.gastro.2012.08.002

3. Tillotson GS, Tillotson J (2011) Clostridium difficile-a moving target. F1000 Med Rep 3:6. https://doi.org/10.3410/M3-6

4. DePestel DD, Aronoff DM (2013) Epidemiology of Clostridium difficile infection. J Pharm Pract 26:464-475. https://doi.org/10. 1177/0897190013499521

5. Eyre DW, Cule ML, Wilson DJ, Griffiths D, Vaughan A, O'Connor L, Ip CLC, Golubchik T, Batty EM, Finney JM, Wyllie DH, Didelot X, Piazza P, Bowden R, Dingle KE, Harding RM, Crook DW, Wilcox MH, Peto TEA, Walker AS (2013) Diverse sources of $C$. difficileinfection identified on whole-genome sequencing. N Engl J Med 369:1195-1205. https://doi.org/10.1056/NEJMoa1216064

6. Vincent C, Miller MA, Edens TJ, Mehrotra S, Dewar K, Manges AR (2016) Bloom and bust: intestinal microbiota dynamics in response to hospital exposures and Clostridium difficile colonization or infection. Microbiome 4:12. https://doi.org/10.1186/s40168-016-0156-3

7. Gianotti RJ, Moss AC (2017) Fecal microbiota transplantation: from Clostridium difficile to inflammatory bowel disease. Gastroenterol Hepatol (N Y) 13:209-213

8. Kelly CP (2013) Fecal microbiota transplantation-an old therapy comes of age. N Engl J Med 368:474-475. https://doi.org/10.1056/ NEJMe1214816
9. Collins MD, Lawson PA, Willems A, Cordoba JJ, FernandezGarayzabal J, Garcia P, Cai J, Hippe H, Farrow JA (1994) The phylogeny of the genus Clostridium: proposal of five new genera and eleven new species combinations. Int J Syst Bacteriol 44:812 826. https://doi.org/10.1099/00207713-44-4-812

10. Gupta RS, Gao B (2009) Phylogenomic analysis of clostridia and identification of novel protein signatures that are specific to the genus Clostridium sensu stricto (cluster I). Int J Syst Evol Microbiol 59:285-294. https://doi.org/10.1099/ijs.0.001792-0

11. Lawson PA, Rainey FA (2016) Proposal to restrict the genus Clostridium Prazmowski to Clostridium butyricum and related species. Int J Syst Evol Microbiol 66:1009-1016. https://doi.org/10. 1099/ijsem.0.000824

12. Lawson PA, Citron DM, Tyrrell KL, Finegold SM (2016) Reclassification of Clostridium difficile as Clostridioides difficile (hall and O'Toole 1935) Prevot 1938. Anaerobe 40:95-99. https:// doi.org/10.1016/j.anaerobe.2016.06.008

13. Monot M, Eckert C, Lemire A, Hamiot A, Dubois T, Tessier C, Dumoulard B, Hamel B, Petit A, Lalande V, Ma L, Bouchier C, Barbut F, Dupuy B (2015) Clostridium difficile: new insights into the evolution of the pathogenicity locus. Sci. Rep. 5:15023. https:// doi.org/10.1038/srep15023

14. Dingle KE, Elliott B, Robinson E, Griffiths D, Eyre DW, Stoesser N, Vaughan A, Golubchik T, Fawley WN, Wilcox MH, Peto TE, Walker AS, Riley TV, Crook DW, Didelot X (2014) Evolutionary history of the Clostridium difficile pathogenicity locus. Genome Biol Evol 6:36-52. https://doi.org/10.1093/gbe/evt204

15. Brouwer MSM, Roberts AP, Hussain H, Williams RJ, Allan E, Mullany P (2013) Horizontal gene transfer converts non-toxigenic Clostridium difficile strains into toxin producers. Nat Commun 4: 2601. https://doi.org/10.1038/ncomms3601

16. Stiles BG, Wigelsworth DJ, Popoff MR, Barth H (2011) Clostridial binary toxins: iota and $\mathrm{C} 2$ family portraits. Front Cell Infect Microbiol 1:11. https://doi.org/10.3389/fcimb.2011.00011

17. Cowardin CA, Buonomo EL, Saleh MM, Wilson MG, Burgess SL, Kuehne SA, Schwan C, Eichhoff AM, Koch-Nolte F, Lyras D, Aktories K, Minton NP, Petri Jr WA (2016) The binary toxin CDT enhances Clostridium difficile virulence by suppressing protective colonic eosinophilia. Nat Microbiol 1:16108. https://doi.org/ 10.1038/nmicrobiol.2016.108

18. Jia H, Du P, Yang H, Zhang Y, Wang J, Zhang W, Han G, Han N, Yao Z, Wang H, Zhang J, Wang Z, Ding Q, Qiang Y, Barbut F, Gao GF, Cao Y, Cheng Y, Chen C (2016) Nosocomial transmission of Clostridium difficile ribotype 027 in a Chinese hospital, 20122014, traced by whole genome sequencing. BMC Genomics 17: 405. https://doi.org/10.1186/s12864-016-2708-0

19. Knight DR, Elliott B, Chang BJ, Perkins TT, Riley TV (2015) Diversity and evolution in the genome of Clostridium difficile. Clin Microbiol Rev 28:721-741. https://doi.org/10.1128/CMR. 00127-14

20. Mac Aogáin M, Moloney G, Kilkenny S, Kelleher M, Kelleghan M, Boyle B, Rogers TR (2015) Whole-genome sequencing improves discrimination of relapse from reinfection and identifies transmission events among patients with recurrent Clostridium difficile infections. J Hosp Infect 90:108-116. https://doi.org/10. 1016/j.jhin.2015.01.021

21. Culligan EP, Sleator RD (2016) Advances in the microbiome: applications to Clostridium difficile infection. J Clin Med 5(9). doi: https://doi.org/10.3390/jcm5090083

22. Caroff DA, Yokoe DS, Klompas M (2017) Evolving insights into the epidemiology and control of Clostridium difficile in hospitals. Clin Infect Dis. https://doi.org/10.1093/cid/cix456

23. Roy Chowdhury P, DeMaere M, Chapman T, Worden P, Charles IG, Darling AE, Djordjevic SP (2016) Comparative genomic analysis of toxin-negative strains of Clostridium difficile from humans 
and animals with symptoms of gastrointestinal disease. BMC Microbiol 16:41. https://doi.org/10.1186/s12866-016-0653-3

24. Mahato NK, Gupta V, Singh P, Kumari R, Verma H, Tripathi C, Rani P, Sharma A, Singhvi N, Sood U, Hira P, Kohli P, Nayyar N, Puri A, Bajaj A, Kumar R, Negi V, Talwar C, Khurana H, Nagar S, Sharma M, Mishra H, Singh AK, Dhingra G, Negi RK, Shakarad M, Singh Y, Lal R (2017) Microbial taxonomy in the era of OMICS: application of DNA sequences, computational tools and techniques. Antonie Van Leeuwenhoek. https://doi.org/10.1007/ s10482-017-0928-1

25. Konstantinidis KT, Tiedje JM (2005) Towards a genome-Bbased taxonomy for prokaryotes. J Bacteriol 187:6258-6264. https://doi. org/10.1128/JB.187.18.6258-6264.2005

26. Thompson CC, Chimetto L, Edwards RA, Swings J, Stackebrandt E, Thompson FL (2013) Microbial genomic taxonomy. BMC Genomics 14:913-913. https://doi.org/10.1186/1471-2164-14-913

27. Jun SR, Wassenaar TM, Nookaew I, Hauser L, Wanchai V, Land M, Timm CM, Lu TY, Schadt CW, Doktycz MJ, Pelletier DA, Ussery DW (2015) Diversity of Pseudomonas genomes, including Populus-associated isolates, as revealed by comparative genome analysis. Appl Environ Microbiol 82:375-383. https://doi.org/10. 1128/aem.02612-15

28. Edgar RC Search and clustering orders of magnitude faster than BLAST. Bioinformatics 26:2460-2461. https://doi.org/10.1093/ bioinformatics/btq461

29. Gascuel O (1997) BIONJ: an improved version of the NJ algorithm based on a simple model of sequence data. Mol Biol Evol 14:685-695

30. Wanchai V, Patumcharoenpol P, Nookaew I, Ussery D (2017) dBBQs: dataBase of bacterial quality scores. BMC Bioinf 18:483. https://doi.org/10.1186/s12859-017-1900-9

31. Varghese NJ, Mukherjee S, Ivanova N, Konstantinidis KT, Mavrommatis K, Kyrpides NC, Pati A (2015) Microbial species delineation using whole genome sequences. Nucleic Acids Res 43: 6761-6771. https://doi.org/10.1093/nar/gkv657

32. Edgar RC (2004) MUSCLE: a multiple sequence alignment method with reduced time and space complexity. BMC Bioinf 5:113. https://doi.org/10.1186/1471-2105-5-113

33. Hyatt D, Chen GL, Locascio PF, Land ML, Larimer FW, Hauser LJ (2010) Prodigal: prokaryotic gene recognition and translation initiation site identification. BMC Bioinf 11:119. https://doi.org/10. 1186/1471-2105-11-119

34. Johnson LS, Eddy SR, Portugaly E (2010) Hidden Markov model speed heuristic and iterative HMM search procedure. BMC Bioinf 11:431. https://doi.org/10.1186/1471-2105-11-431

35. Finn RD, Coggill P, Eberhardt RY, Eddy SR, Mistry J, Mitchell AL, Potter SC, Punta M, Qureshi M, Sangrador-Vegas A, Salazar GA, Tate J, Bateman A (2016) The Pfam protein families database: towards a more sustainable future. Nucleic Acids Res. 44:D279D285. https://doi.org/10.1093/nar/gkv1344

36. Milani C, Ticinesi A, Gerritsen J, Nouvenne A, Lugli GA, Mancabelli L, Turroni F, Duranti S, Mangifesta M, Viappiani A, Ferrario C, Maggio M, Lauretani F, De Vos W, van Sinderen D, Meschi T, Ventura M (2016) Gut microbiota composition and Clostridium difficile infection in hospitalized elderly individuals: a metagenomic study. Sci Rep 6:25945. https://doi.org/10.1038/srep25945

37. Li H, Durbin R (2009) Fast and accurate short read alignment with burrows-wheeler transform. Bioinformatics 25:1754-1760. https:// doi.org/10.1093/bioinformatics/btp324

38. Quinlan AR, Hall IM (2010) BEDTools: a flexible suite of utilities for comparing genomic features. Bioinformatics 26:841-842. https://doi.org/10.1093/bioinformatics/btq033

39. Robinson JT, Thorvaldsdottir H, Winckler W, Guttman M, Lander ES, Getz G, Mesirov JP (2011) Integrative genomics viewer. Nat Biotechnol 29:24-26. https://doi.org/10.1038/nbt.1754
40. Bae JW, Park JR, Chang YH, Rhee SK, Kim BC, Park YH (2004) Clostridium hastiforme is a later synonym of Tissierella praeacuta. Int J Syst Evol Microbiol 54:947-949. https://doi. org/10.1099/ijs.0.63068-0

41. Vesth T, Ozen A, Andersen SC, Kaas RS, Lukjancenko O, Bohlin J, Nookaew I, Wassenaar TM, Ussery DW (2013) Veillonella, Firmicutes: microbes disguised as gram negatives. Stand Genomic Sci 9:431-448. https://doi.org/10.4056/sigs.2981345

42. Ueda K, Beppu T (2007) Lessons from studies of Symbiobacterium thermophilum, a unique syntrophic bacterium. Biosci Biotechnol Biochem 71:1115-1121. https://doi.org/10.1271/bbb.60727

43. Tu Q, Lin L (2016) Gene content dissimilarity for subclassification of highly similar microbial strains. BMC Genomics 17:647. https:// doi.org/10.1186/s12864-016-2991-9

44. Beaz-Hidalgo R, Hossain MJ, Liles MR, Figueras MJ (2015) Strategies to avoid wrongly labelled genomes using as example the detected wrong taxonomic affiliation for aeromonas genomes in the GenBank database. PLoS One 10(1):e0115813. https://doi. org/10.1371/journal.pone.0115813

45. Eckert C, Coignard B, Hebert M, Tarnaud C, Tessier C, Lemire A, Burghoffer B, Noel D, Barbut F (2013) Clinical and microbiological features of Clostridium difficile infections in France: the ICDRAISIN 2009 national survey. Med Mal Infect 43:67-74. https:// doi.org/10.1016/j.medmal.2013.01.004

46. Kurka H, Ehrenreich A, Ludwig W, Monot M, Rupnik M, Barbut F, Indra A, Dupuy B, Liebl W (2014) Sequence similarity of Clostridium difficile strains by analysis of conserved genes and genome content is reflected by their ribotype affiliation. PLoS One 9:e86535. https://doi.org/10.1371/journal.pone.0086535

47. NIH HMP Working Group, Peterson J, Garges S, Giovanni M, McInnes P, Wang L, Schloss JA, Bonazzi V, McEwen JE, Wetterstrand KA, Deal C, Baker CC, Di Francesco V, Howcroft TK, Karp RW, Lunsford RD, Wellington CR, Belachew T, Wright M, Giblin C, David H, Mills M, Salomon R, Mullins C, Akolkar B, Begg L, Davis C, Grandison L, Humble M, Khalsa J, Little AR, Peavy H, Pontzer C, Portnoy M, Sayre MH, Starke-Reed P, Zakhari S, Read J, Watson B, Guyer M (2009) The NIH human microbiome project. Genome Res. 19:2317-2323. https://doi.org/10.1101/gr.096651.109

48. He M, Sebaihia M, Lawley TD, Stabler RA, Dawson LF, Martin MJ, Holt KE, Seth-Smith HM, Quail MA, Rance R, Brooks K, Churcher C, Harris D, Bentley SD, Burrows C, Clark L, Corton C, Murray V, Rose G, Thurston S, van Tonder A, Walker D, Wren BW, Dougan G, Parkhill J (2010) Evolutionary dynamics of Clostridium difficile over short and long time scales. Proc Natl Acad Sci U S A 107:7527-7532. https://doi.org/10.1073/pnas.0914322107

49. Maiden MC (2006) Multilocus sequence typing of bacteria. Annu Rev Microbiol 60:561-588. https://doi.org/10.1146/annurev.micro. 59.030804 .121325

50. Zolfo M, Tett A, Jousson O, Donati C, Segata N (2017) MetaMLST: multi-locus strain-level bacterial typing from metagenomic samples. Nucleic Acids Res 45:e7. https://doi.org/ 10.1093/nar/gkw837

51. Rupnik M, Brazier JS, Duerden BI, Grabnar M, Stubbs SL (2001) Comparison of toxinotyping and PCR ribotyping of Clostridium difficile strains and description of novel toxinotypes. Microbiology 147:439-447. https://doi.org/10.1099/00221287$147-2-439$

52. Darling AE, Worden P, Chapman TA, Roy Chowdhury P, Charles IG, Djordjevic SP (2014) The genome of Clostridium difficile 5.3. Gut Pathog 6(1):4. https://doi.org/10.1186/1757-4749-6-4

53. Wasels F, Barbanti F, Spigaglia P (2016) Draft genome sequence of Clostridium difficile strain IT1118, an epidemic isolate belonging to the emerging PCR ribotype 018. Genome Announc 4(4). https:// doi.org/10.1128/genomeA.00717-16 